\title{
Genetic dissection of milk yield traits and mastitis resistance quantitative trait loci on chromosome 20 in dairy cattle ${ }^{1}$
}

\author{
Naveen K. Kadri, Bernt Guldbrandtsen, Mogens S. Lund, and Goutam Sahana ${ }^{2}$ \\ Center for Quantitative Genetics and Genomics, Department of Molecular Biology and Genetics, Aarhus University, 8830 Tjele, Denmark
}

\begin{abstract}
Intense selection to increase milk yield has had negative consequences for mastitis incidence in dairy cattle. Due to low heritability of mastitis resistance and an unfavorable genetic correlation with milk yield, a reduction in mastitis through traditional breeding has been difficult to achieve. Here, we examined quantitative trait loci (QTL) that segregate for clinical mastitis and milk yield on Bos taurus autosome 20 (BTA20) to determine whether both traits are affected by a single polymorphism (pleiotropy) or by multiple closely linked polymorphisms. In the latter but not the former situation, undesirable genetic correlation could potentially be broken by selecting animals that have favorable variants for both traits. First, we performed a within-breed association study using a haplotype-based method in Danish Holstein cattle (HOL). Next, we analyzed Nordic Red dairy cattle (RDC) and Danish Jersey cattle (JER) with the goal of determining whether these QTL identified in Holsteins were segregating across breeds. Genotypes for 12,566 animals (5,966 HOL, 5,458 RDC, and 1,142 JER) were determined by using the Illumina Bovine SNP50 BeadChip (50K; Illumina, San Diego, $\mathrm{CA}$ ), which identifies 1,568 single nucleotide polymorphisms on BTA20. Data were combined, phased, and clustered into haplotype states, followed by within- and across-breed haplotype-based association analyses using a linear mixed model. Association signals for both clinical mastitis and milk yield peaked in the 26- to 40$\mathrm{Mb}$ region on BTA20 in HOL. Single-variant association analyses were carried out in the QTL region using whole sequence level variants imputed from references of 2,036 HD genotypes (BovineHD BeadChip; Illumina) and 242 whole-genome sequences. The milk QTL were also segregating in RDC and JER on the BTA20targeted region; however, an indication of differences in the causal factor(s) was observed across breeds. A pre-
\end{abstract}

Received March 20, 2015.

Accepted July 25, 2015.

${ }^{1}$ Part of the PhD study of Naveen K. Kadri.

${ }^{2}$ Corresponding author: goutam.sahana@mbg.au.dk viously reported F279Y mutation (rs385640152) within the growth hormone receptor gene showed strong association with milk, fat, and protein yields. In HOL, the highest peaks for milk yield and susceptibility to mastitis were separated by over $3.5 \mathrm{Mb}(3.8 \mathrm{Mb}$ by haplotype analysis, $3.6 \mathrm{Mb}$ by single nucleotide polymorphism analysis), suggesting separate genetic variants for the traits. Further analysis yielded 2 candidate mutations for the mastitis QTL, at 33,642,072 bp (rs378947583) in an intronic region of the caspase recruitment domain protein 6 gene and 35,969,994 bp (rs133596506) in an intronic region of the leukemia-inhibitory factor receptor gene. These findings suggest that it may be possible to separate these beneficial and detrimental genetic factors through targeted selective breeding.

Key words: clinical mastitis, association study, milk yield, cattle

\section{INTRODUCTION}

Intense selection to increase milk yield and milk quality in dairy cows has had negative consequences on other functional traits, including the incidence of clinical mastitis (CM; Oltenacu and Broom, 2010). Attempts to improve CM resistance through traditional breeding have not been very successful due to low heritability estimates for CM and unfavorable genetic correlations between CM resistance and milk yield (Lund et al., 1999; Heringstad et al., 2000; Heringstad et al., 2005). Therefore, CM remains the most frequent and costly disease in dairy production, affecting $18 \%$ of all dairy cows (USDA, 2008). Several studies from Nordic countries have reported QTL for CM (Lund et al., 2007; Sahana et al., 2008, 2013; Schulman et al., 2009, 2011; Sodeland et al., 2011). Linkage disequilibrium (LD) spreads across large chromosomal regions, especially in Holsteins (de Roos et al., 2008), making it difficult to distinguish the quantitative trait nucleotide from SNP that are in strong LD with the causal variants. Thus, the reported QTL intervals are broad, impeding identification of affected genes and causal polymorphisms. However, the availability of whole-genome sequencing (WGS) data for dairy cattle has increased the chance 
of identifying causative polymorphisms underlying QTL. Availability of multiple breed data may help in breaking the long stretch of strong LD.

Many economically important traits, including milk yield and CM, are polygenic. As a result, variance of these traits may result from the interaction of small changes in the activity of multiple, potentially neighboring, genes that interact and affect the trait in question, making it very challenging to identify individual genes. Single QTL can act through multiple pathways and can affect not only coding regions, but also regulatory regions flanking these genes (Bickel et al., 2011). In recent years, genomic prediction approaches that simultaneously incorporate information from thousands of genetic markers to predict the genetic merit of an animal have come into routine use in dairy cattle breeding (Lund et al., 2011). However, current breeding techniques have not been effective in disrupting unfavorable genetic correlations between $\mathrm{CM}$ and milk yield. One factor that has contributed to this problem is the lack of sufficiently detailed genetic information in the models to clarify whether both traits are affected by a single polymorphism (pleiotropy) or by multiple closely linked polymorphisms. Besides, the recognition of correlations between traits depends on the identification of genetic covariance: that is, the gene frequencies of loci contributing positively and negatively to covariance (Bohren et al., 1966; van Binsbergen et al., 2012). Current prediction methods could be improved by incorporating information on important identified causative genetic variants (Brøndum et al., 2015; Zhang et al., 2015).

Sahana et al. (2014) recently reported a QTL on BTA20 affecting CM and SCS in Danish Holstein cattle (HOL), with a broad association signal at 32 to 40 $\mathrm{Mb}$ and a peak around $35 \mathrm{Mb}$. In a meta-analysis of 3 breeds [HOL, Nordic Red dairy cattle (RDC), and Danish Jersey cattle (JER)], the most highly associated SNP with CM was located at 33,398,781 bp (Sahana et al., 2014). Those authors also reported strong evidence for a QTL located near $33 \mathrm{Mb}$ on BTA20 for milk yield, milk fat, and milk protein. Finally, an F279Y mutation (rs385640152) in the growth hormone receptor (GHR) gene at 31,909,478 bp has been reported to affect milk, fat, protein, and SCS in HOL (Blott et al., 2003; Rahmatalla et al., 2011).

The large number of reports describing QTL that affect both milk yield and CM susceptibility traits led us to target this region of BTA20 for further study. We hoped to determine whether mutations in a single gene affected both CM and milk traits, or whether multiple causal factors segregated in Nordic cattle breeds that may be separable by more detailed analysis. Our objectives were (1) to fine-map QTL for CM and milk traits (milk yield, milk fat, and milk protein) on BTA20 using imputed WGS variants incorporating data from 3 dairy breeds, and (2) to determine whether QTL for CM and milk traits on BTA20 were due to different but closely linked polymorphisms or to multiple effects of the same polymorphism.

\section{MATERIALS AND METHODS}

\section{Animal Populations and Phenotypes}

Data of 12,566 genotyped bulls from 3 Nordic dairy cattle breeds (5,966 HOL, 5,458 RDC, and 1,142 JER) and their EBV for 4 indices-CM and milk, fat, and protein yields - were used in the study. The EBV for milk, fat, and protein yields (in kilograms) were estimated by Nordic Cattle Genetic Evaluation (http:// www.nordicebv.info) based on production figures derived from routine test-day records and converted to an index for each trait by using a random regression test-day model. Data included are test-day records from d 8 to 365 for first to third lactations. Estimation of EBV for CM was described previously by Sahana et al. (2014). Mean reliabilities for EBV were 0.79 for CM and 0.92 for milk yield traits, respectively. Seventy-five percent of individuals had reliability for CM above 0.76 and above 0.92 for the 3 milk yield indices.

\section{SNP Chip and Genotyping}

All animals were individually genotyped by using the Bovine SNP50 BeadChip (Illumina Inc., San Diego, CA), which assays for approximately 54,000 SNP markers, 1,568 of which are on BTA20. Genotyping was done at Aarhus University (Foulum, Denmark) and GenoSkan A/S (Tjele, Denmark). Quality parameters used for selection of SNP in the study were minimum call rates of $90 \%$ for animals and $95 \%$ for SNP. The minimum accepted GenCall score (Illumina) was 0.60 for individual typings. Individuals with average GenCall scores below 0.65 were excluded. The SNP positions within a chromosome were defined according to the Bos taurus genome UMD3.1 assembly (Zimin et al., 2009). After quality control analysis, 46,702 SNP were identified on 29 autosomes, with 1,301 on BTA20.

\section{Haplotype Phasing}

Genotype phasing and haplotype clustering were done in a multibreed combined data set, which consisted of genotypes for 12,566 animals for 1,301 SNP remaining after quality control on BTA20. The SNP genotypes were first phased by utilizing information from the pedigree with LINKPHASE and then by using LD with DAGPHASE. Phased genotypes were clustered into 50 
ancestral haplotypes by using HIDDENPHASE. All programs were available in the PHASEBOOK software package (Druet and Georges, 2010).

\section{WGS Data}

Reference population sequences used for imputation of Nordic animals consisted of the WGS data available at the Center for Quantitative Genetics and Genomics, Aarhus University, and through the 1,000 Bull Genomes Project (Daetwyler et al., 2014). Sequences from Aarhus University were aligned to the UMD3.1 assembly of the cattle genome (Zimin et al., 2009) by using bwa (Li and Durbin, 2009). They were converted to raw BAM files by using SAMtools (Li et al., 2009). Quality scores were recalibrated by using the Genome Analysis Toolkit (McKenna et al., 2010) following the Human 1000 Genome guidelines, incorporating information from SNP database (dbSNP; Sherry et al., 2001). Sequences were realigned around insertion/deletions (indel) by using the Genome Analysis Toolkit. Variants were called by using UnifiedGenotyper from the Genome Analysis Toolkit.

Genomes from the 1,000 Bull Genomes Project were sequenced in several laboratories and were analyzed in the Department of Primary Industries (Victoria, Australia). Sequences were aligned to the same reference genome as used at Aarhus University by using bwa. Variant calling was done by using the mpileup function in SAMtools. Variant call files from Aarhus University and the 1,000 Bull Genomes Project run2 were combined by using CombineVariants from the Genome Analysis Toolkit, using calls from the Nordic data set for animals appearing in both data sets. Only polymorphisms identified in both the Aarhus University and 1,000 Bull Genomes data sets were imputed. When both an SNP and an indel were identified at a position, the indel was deleted. The SNP at positions where the alleles between HD and sequence data differed were deleted.

\section{Imputation of High-Density SNP Array Data to the Full Sequence}

A reference population for high-density (HD) genotypes (BovineHD BeadChip; Illumina) was available for 2,036 bulls, consisting of 902 HOL, $735 \mathrm{RDC}$, and 399 JER (Lund et al., 2011). The genotyping procedure and quality control criteria were the same as described above for the 50K SNP array. Imputation of 50K SNP to the full genomic sequence was done in 2 steps. First, $50 \mathrm{~K}$ genotypes (46,702 SNP after quality control) for the 12,566 Nordic bulls were imputed to HD genotypes by using the IMPUTE2 software package (Howie et al., 2009), yielding 648,219 SNP after imputation.

Second, data from the 12,566 bulls were imputed to the whole-genome level. Whole-genome sequences from 242 dairy cattle (132 HOL, 42 JER, 52 RDC, and 16 Brown-Swiss bulls), originating from a combination of sequences processed at Aarhus University and from the 1,000 Bull Genomes data set, were used as references to impute the HD data to the whole-genome level by using the Beagle version 3.3.2 software package (Browning and Browning, 2009). The total number of polymorphic sites in WGS data was 17.28 million. Reference data were prephased with Beagle v3.3.2 (Browning and Browning, 2009). For this imputation, chromosomes were divided into chunks of about 20,000 consecutive markers with an overlap of 250 markers at each end to minimize imputation errors at the ends of the regions. Details of the imputation procedure and imputation accuracy have been described by Brøndum et al. (2014). After markers with an imputation quality score below 0.9 were removed, 8,938,927 markers remained for BTA1 to 29 with 276,766 SNP on BTA20. The imputed allele dosages were used for association analysis.

\section{Population Structure}

We used sequencing data from HOL for the discovery and fine mapping of QTL and used 2 additional breeds (RDC and JER) as validation populations. Nordic RDC data consisted of combined sequences of 3 RDC cattle populations from Denmark, Sweden, and Finland. We studied the population structure of each breed using principal component (PC) analysis of all 46,702 markers on the 50K chip (Price et al., 2006). The first 4 $\mathrm{PC}$ were fitted as covariates in multibreed association analyses to account for population structure.

\section{Haplotype-Based Association Analysis}

Within- and across-breed analyses were carried out for HOL, RDC, and JER by using a haplotype-based analysis with 50K SNP array data. The presence of a QTL for the trait of interest was tested by fitting the haplotype state at each SNP successively into the following model:

$$
\mathbf{y}=\mathbf{1} \mu+\mathbf{X b}+\mathbf{Z}_{\mathbf{h}} \mathbf{h}+\mathbf{Z}_{\mathbf{u}} \mathbf{u}+\mathbf{P c}+\mathbf{e},
$$

where $\mathbf{y}$ is the vector of EBV for the trait of interest; $\mathbf{1}$ is a vector of $1 \mathrm{~s} ; \mu$ is the overall mean; $\mathbf{X}$ is a design matrix assigning animals to breed; $\mathbf{b}$ is a vector of fixed breed effects (only fitted for across-breed analysis); $\mathbf{h}$ is a vector of random QTL effects $\mathbf{h} \sim N\left(0, \mathbf{I} \sigma_{H}^{2}\right)$ whose 
elements are the effects of the 50 reconstructed haplotypes, where $\mathbf{I}$ is the identity matrix, $\sigma_{H}^{2}$ is the haplotype variance, $\mathbf{u}$ is the vector of individual polygenic effects with variance $\sigma_{g}^{2}$, with $\mathbf{u} \sim N\left(0, \mathbf{A} \sigma_{g}^{2}\right)$, where $\mathbf{A}$ is the additive relationship matrix estimated from the pedigree, and $\sigma_{g}^{2}$ is the polygenic variance; $\mathbf{Z}_{\mathbf{h}}$ and $\mathbf{Z}_{\mathbf{u}}$ are design matrices related to the haplotypes and polygenic effects; $\mathbf{P}$ is the matrix of the loadings on the first $4 \mathrm{PC}$ estimated from the genome-wide markers; $\mathbf{c}$ is the vector of regression coefficients of $\mathrm{PC}$; and $\mathbf{e}$ is the vector of independent individual residual terms with variance $\sigma_{\mathbf{e}}^{2}$, assumed to be $\mathbf{e} \sim N\left(0, \mathbf{I} \sigma_{e}^{2}\right)$. Variances $\sigma_{H}^{2}, \sigma_{\mathbf{e}}^{2}$, and $\sigma_{g}^{2}$ were estimated by using AI-REML with DMU software (Madsen and Jensen, 2010).

Haplotype effect at a given position was tested with a likelihood ratio test comparing the haplotype-based model with the null model (without the haplotype effect). Under the null hypothesis, the test statistic has a $\chi^{2}$ distribution with 1 degree of freedom. No PC was fitted for within-breed analysis. Determination of the significance for each association was based on genomewide Bonferroni multiple testing correction for the total number of SNP because the haplotype states were derived for each SNP. Therefore, the significance threshold was at $P<1.07 \times 10^{-6}$ (5.97 on a $-\log _{10}$ scale). We added the most significantly associated SNP as a cofactor in the haplotype model to examine whether multiple variants associated with the traits were present within the QTL region.

\section{Single-Marker Association Analysis}

Associations between the imputed sequence variants and phenotypes were assessed by a single-locus regression analysis for each SNP separately, using a linear mixed model ( $\mathrm{Yu}$ et al., 2006). Association analyses were carried out by successively fitting the imputed genotype doses at each variant in a linear mixed model with random individual effects. The model was as follows:

$$
\mathbf{y}=\mathbf{1} \mu+\mathbf{X b}+m g+\mathbf{Z} \mathbf{u}+\mathbf{P c}+\mathbf{e},
$$

where $\mathbf{y}$ is the vector of phenotypes (EBV); $\mathbf{1}$ is a vector of 1s with length equal to the number of observations; $\mu$ is the general mean; $\mathbf{X}$ is a design matrix assigning animals to breeds; $\mathbf{b}$ is a vector of fixed breed effects (only fitted for across-breed analysis); $m$ is the genotypic doses (varying between 0 and 2 ); $g$ is the scalar value of the additive fixed allele substitution effect at the SNP; $\mathbf{Z}$ is an incidence matrix relating phenotypes to the corresponding random polygenic effects; $\mathbf{u}$ is a vector of the random polygenic effects with a multivariate normal distribution, $\mathbf{u} \sim N\left(0, \mathbf{A} \sigma_{g}^{2}\right)$, where $\mathbf{A}$ is the additive relationship matrix and $\sigma_{g}^{2}$ is the polygenic variance; $\mathbf{P}$ is the matrix of loadings on the first $4 \mathrm{PC}$, estimated from the genome-wide markers; $\mathbf{c}$ is the vector of regression coefficients of $\mathrm{PC}$; and $\mathbf{e}$ is a vector of independent individual residual terms with a normal distribution $\mathbf{e} \sim N\left(0, \mathbf{I} \sigma_{\mathbf{e}}^{2}\right)$, where $\sigma_{\mathbf{e}}^{2}$ is the residual variance. No PC was fitted for within-breed analyses. The model was fitted and analyzed by using DMU (Madsen and Jensen, 2010). The standard error of the fixed effect estimates was obtained from DMU. The presence of an effect of a marker was tested by a $t$-test against a null hypothesis, $\mathrm{H}_{0}: g=0$.

\section{RESULTS AND DISCUSSION}

\section{QTL for CM and Milk, Protein, and Fat Yields on BTA20 in HOL}

To understand the genetic architecture of BTA20 that contributes to variation in multiple traits in HOL, we performed an association study for milk, protein, and fat yields and CM using a haplotype-based method. The results are shown in Figure 1A to D (solid line), and the QTL peak positions are listed in Table 1. Significant associations were observed for all tested traits, with milk yield being the most significant and protein yield being the least. The strength of association signals across all traits peaked in the $\sim 28-$ to $36-\mathrm{Mb}$ region (Supplementary Figure S1; http://dx.doi.org/10.3168/ jds.2015-9599). The highest QTL peaks for milk yield and CM were at locations $3.8 \mathrm{Mb}$ apart (Table 1), but QTL peaks for milk and fat yields were located close together $(0.78 \mathrm{Mb})$.

\section{Association with Imputed WGS Variants in HOL}

We took advantage of the available imputed sequence variants to fine-map the QTL affecting milk, protein, and fat yields and CM. Results of single SNP analyses are presented in Figure 1A to D (gray dots). Locations and $-\log _{10}(P)$ of the most significant SNP are listed in Table 1. Again, the most significant association signal was found for milk yield followed by fat yield, CM, and protein yield. The distance between the most significant SNP for milk yield and CM was $3.63 \mathrm{Mb}$, for milk yield and fat yield was $2.66 \mathrm{Mb}$, and for milk yield and protein yield was $1.06 \mathrm{Mb}$.

\section{Most Significant Variants Partly Explain the QTL Variance in HOL}

We observed that, in the single-variant association, the QTL regions were subdivided into multiple peaks 
Table 1. Peaks of the association signal and $-\log _{10}(P)$ for the haplotype-based analysis with the Bovine SNP50 BeadChip (Illumina, San Diego, CA) array and single-marker analysis using whole-genome SNP polymorphisms within the targeted region on BTA20 in Holstein cattle

\begin{tabular}{|c|c|c|c|c|}
\hline \multirow[b]{2}{*}{ Trait } & \multicolumn{2}{|c|}{ Haplotype (50K) } & \multicolumn{2}{|c|}{$\begin{array}{l}\text { Single marker analysis } \\
\text { (imputed sequence) }\end{array}$} \\
\hline & Position (bp) & $-\log _{10}(P)$ & Position (bp) & $-\log _{10}(P)$ \\
\hline Mastitis & $35,102,203$ & 13.08 & $33,642,072$ & 14.69 \\
\hline Milk & $31,293,350$ & 17.42 & $30,005,316$ & 15.40 \\
\hline Fat & $32,074,342$ & 12.50 & $32,663,326$ & 15.16 \\
\hline Protein & $28,773,049$ & 8.51 & $31,061,701$ & 9.22 \\
\hline
\end{tabular}

for all 4 traits, possibly indicating that multiple QTL segregate for each trait in HOL (Figure 1A-D). To determine whether the most significant SNP explain the entire QTL variance for the targeted region, we fitted the most significant SNP variant from the single-marker analysis as a cofactor and repeated the haplotype-based analyses for all 4 traits in HOL (Figure 2A-D).
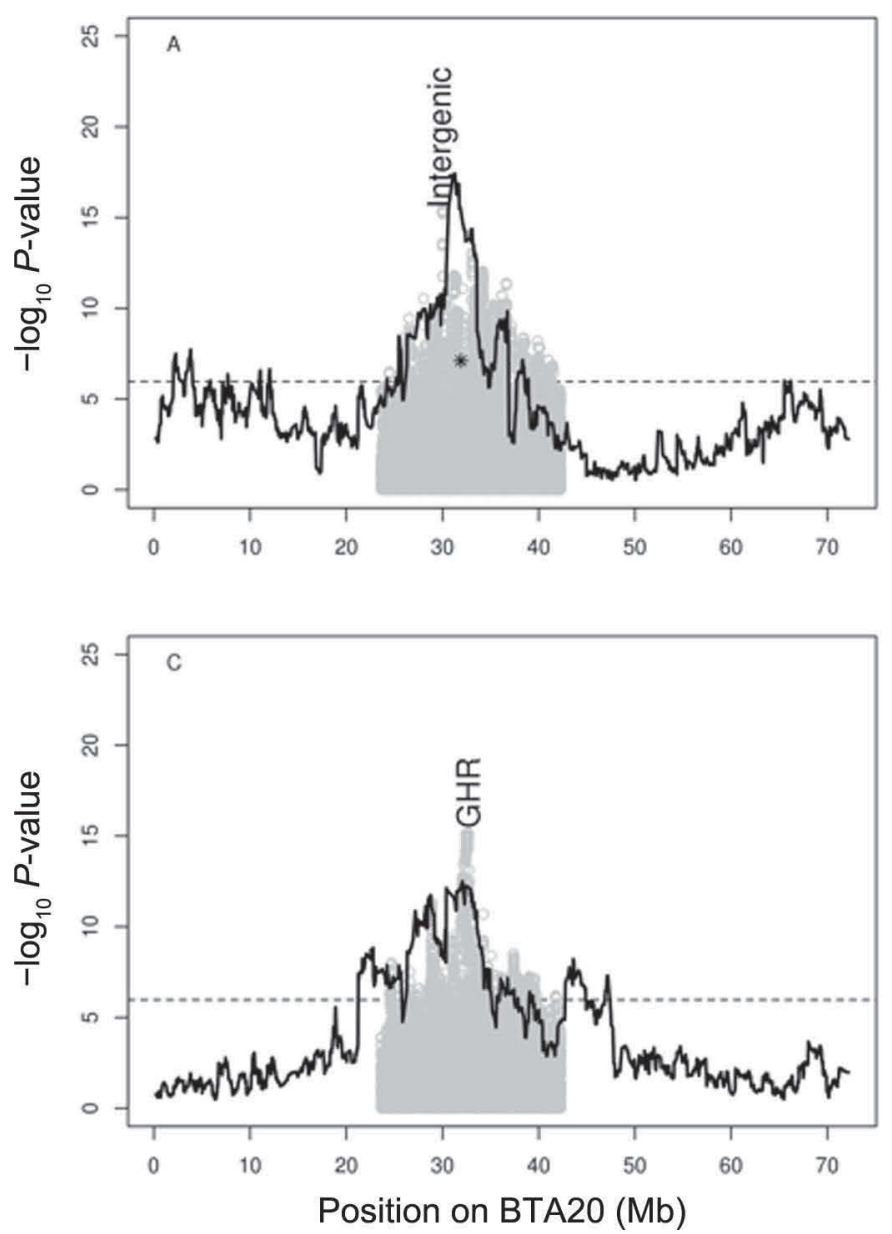

Milk Yield. The most significant variant for milk yield was located in the intergenic region of BTA20 at $30,005,316 \mathrm{bp}$ [rs43140725, minor allelic frequency $(\mathbf{M A F})=0.11]$. Upon fitting the association model, this variant did not fully annihilate the signal obtained with the haplotypes (red line in Figure 2A). This observation indicates that multiple causative factors for milk
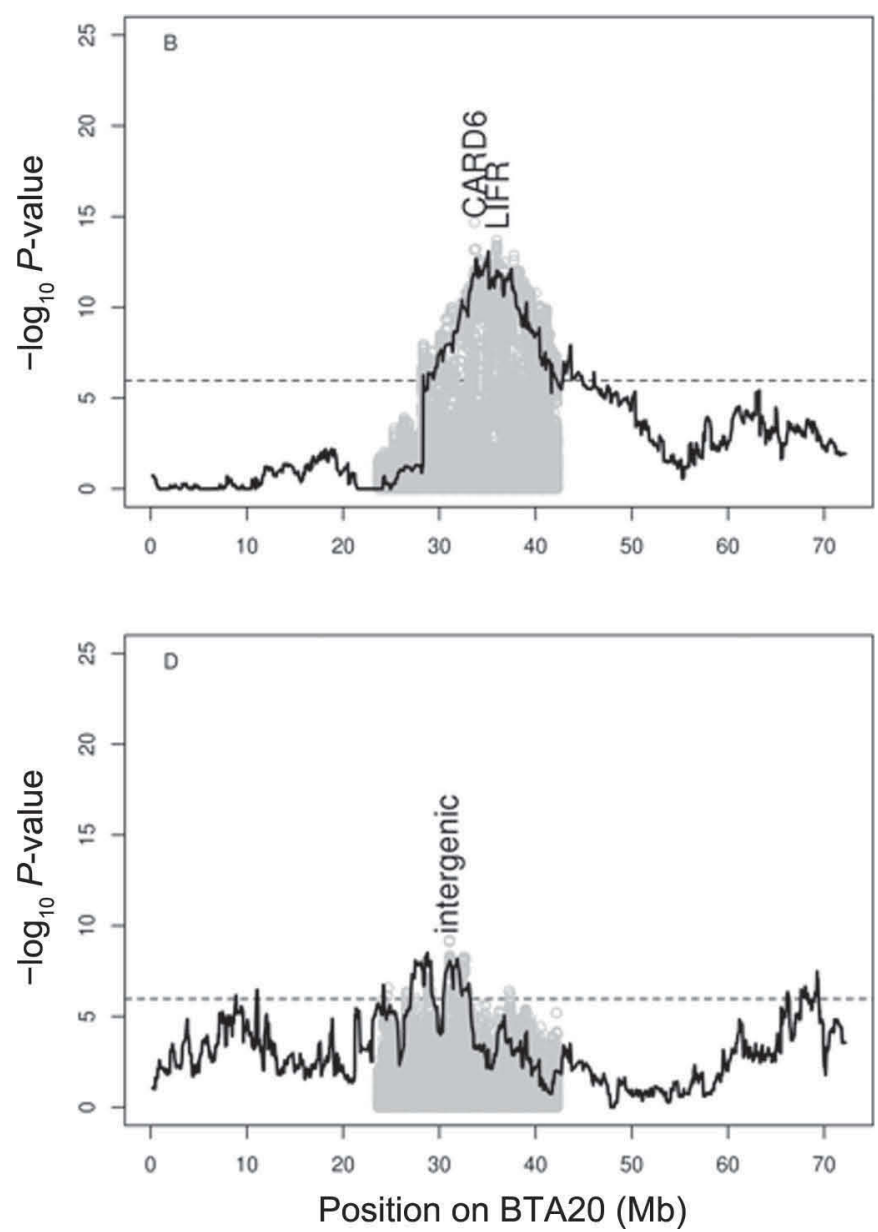

Figure 1. Quantitative trait loci mapping on BTA20 in Nordic Holstein cattle using the haplotype-based method, with QTL data for milk (A), clinical mastitis (B), fat (C), and protein (D). A solid line represents the haplotype-based QTL data. Dotted horizontal lines indicate the Bonferroni-corrected significance threshold. Gray circles represent the results of single variant analysis using the imputed sequence variants in the $\sim 20$ - to $40-\mathrm{Mb}$ region from single-marker regression. 

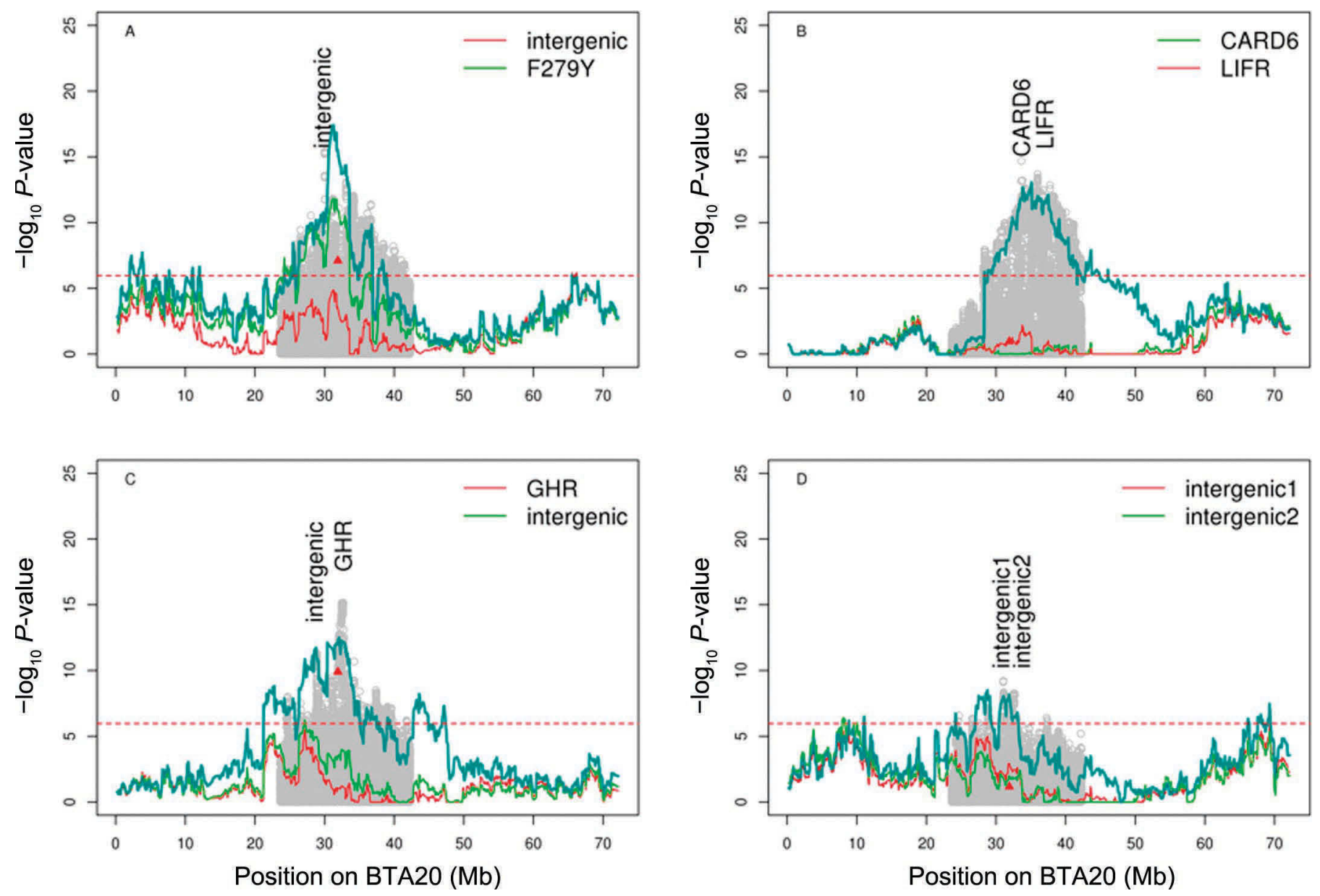

Figure 2. Search for multiple QTL in Holstein cattle by fitting the most significant variant from single-marker analysis in haplotype-based analysis for milk yield (A), clinical mastitis (B), fat yield (C), and protein yield (D). Red triangles are the F279Y mutation. The blue curve indicates the $-\log 10(P)$ value for the haplotype test and the gray dots indicate the test statistics for the single-marker association analysis. The other solid curves indicate the test statistics from the haplotype based analysis with top SNP as cofactors. Dotted horizontal lines (red) indicate the Bonferroni-corrected significance threshold.

yield are probably segregating in HOL. We also tested the effect of the F279Y mutation (rs385640152) in the GHR gene located at $31,909478 \mathrm{bp}$, which was previously reported to have a large effect on milk yield traits (Blott et al., 2003). The F279Y mutation explained less of the QTL variance (green line in Figure 2A) than the intergenic variant at $30,005,316 \mathrm{bp}(\mathrm{rs} 43140725$, red line in Figure 2A).

$\boldsymbol{C M}$. The peak of association for $\mathrm{CM}$ was found at $33,642,072 \mathrm{bp}(\mathrm{rs} 378947583, \mathrm{MAF}=0.23)$, in an intronic region of the caspase recruitment domain $(C A R D)$ family, member 6 gene $(C A R D 6$; ENSBTAG00000014374). The second-highest peak appeared at $35,969,994 \mathrm{bp}(\mathrm{rs} 133596506, \mathrm{MAF}=0.29)$, downstream of the leukemia-inhibitory factor receptor gene (LIFR; ENSBTAG00000010423; downstream gene variant). Fitting either of these 2 variants to the model reduced the association signal below the significance threshold in the haplotype model (Figure 2B). The correlation between the genotype doses of these 2 variants was $0.66(\mathrm{r}=0.45)$.

Fat Yield. The highest peak for fat yield was found at $32,663,326 \mathrm{bp}$ (rs109827679, MAF $=0.24)$, in an intergenic region close to the GHR gene $(31,890,736-$ $32,199,996 \mathrm{bp})$. Fitting this variant partly explained the association signal at the GHR gene (Figure 2C), but the residual signal remained significant, with a peak at $27,170,720$ bp $\left[\mathrm{rs} 110757147,-\log _{10}(P)=5.52\right]$. Similar to milk yield QTL, multiple factors in the targeted region of BTA20 appear to affect fat yield in HOL.

Protein Yield. The first and second most significant variants of protein yield were located at 31,061,701 (rs109176403) and 32,663,326 bp (rs109827679), respectively (Figure 2D). Fitting the variant at 31,061,701 into the model reduced the significance in the GHR gene region to below the threshold. However, a second- 
Table 2. Association peaks for haplotype analyses in the 3 cattle breeds using the Bovine SNP50 BeadChip array (Illumina, San Diego, CA)

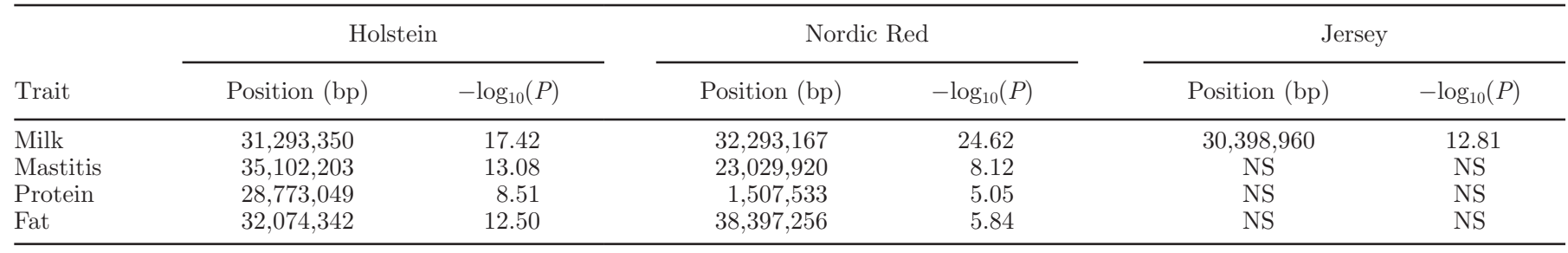

ary suggestive peak at $28.77 \mathrm{Mb}\left[-\log _{10}(P)=5.008\right]$ was close to the Bonferroni-corrected threshold for significance (5.97), providing evidence for segregation of multiple causal factors for protein yield in HOL.

\section{Bivariate Analysis of Milk Yield and CM for Top SNP in HOL}

We analyzed bivariate models for milk yield and $\mathrm{CM}$ with different combinations of the 2 most significant SNP from the single-marker analysis: $\mathrm{SNP}_{\text {milk }}$ at $30,005,316 \mathrm{bp}(\mathrm{rs} 43140725)$ and $\mathrm{SNP}_{\mathrm{CM}}$ at $33,642,072$ bp (rs378947583). These SNP were tested in a bivariate model to determine whether each variant effects only 1 or both of these traits. Models testing the association of $\mathrm{SNP}_{\text {milk }}$ with milk yield and $\mathrm{SNP}_{\mathrm{CM}}$ with $\mathrm{CM}$ had the lowest Akaike and Bayesian information criteria values. Details of the model comparisons and the effect estimates are presented in Supplementary Tables S1 and S2, respectively (http://dx.doi.org/10.3168/jds.20159599). The allele substitution effects clearly indicated that $\mathrm{SNP}_{\text {milk }}$ had a very significant effect on milk yield $\left(P=7.29 \times 10^{-16}\right)$ but not on CM $(P=0.21)$. Similarly, $\mathrm{SNP}_{\mathrm{CM}}$ had a highly significant effect on $\mathrm{CM}(P=$ $\left.1.20 \times 10^{-15}\right)$ but not on milk yield $(P=0.77)$.

\section{Validation of CM and milk QTL in RDC and JER}

We were also interested in whether the QTL for milk traits and $\mathrm{CM}$ discovered in HOL also segregate in RDC and JER. Population structures of these breeds are presented in Supplementary Figure S2 (http:// dx.doi.org/10.3168/jds.2015-9599), which shows that the 3 RDC subpopulations are closely related. A subtle admixture was also observed between HOL and Danish RDC, consistent with the fact that a few HOL bulls were used for breeding in Danish RDC. The JER population appears distinct from the other breeds in this study.

To validate the QTL regions detected in HOL, we carried out within-breed haplotype-based association analyses in RDC and JER for SNP on BTA20 [Supplementary Figure S3 (http://dx.doi.org/10.3168/ jds.2015-9599) and Table 2]. In RDC, significant as- sociation signals were obtained for milk yield and CM (Supplementary Figure S3A), but in JER only the milk yield QTL was significant (Supplementary Figure S3B). We performed a bivariate model analysis to examine if the milk yield haplotype also affected $\mathrm{CM}$ in RDC (Supplementary Table S3; http://dx.doi.org/10.3168/ jds.2015-9599). The haplotype only affected milk yield. The milk yield QTL was significant in all 3 breeds, and the signals approximately overlapped in a $2-\mathrm{Mb}$ region (Table 2).

\section{Combined Breed Analysis for Milk Yield QTL on BTA20}

We identified QTL for milk yield that segregated in approximately the same region $(30.3-32.3 \mathrm{Mb})$ across all 3 breeds individually. Therefore, we repeated the association analysis for milk in the across-breed combined data set. Haplotypes were jointly inferred in 3 breeds, and a haplotype-based analysis was carried out in BTA20. We obtained a highly significant QTL at $32,293,167$ bp (rs41639265, Figure 3). Next, we performed the single-marker analyses in the $28-$ to $40-\mathrm{Mb}$ targeted region using the imputed WGS variants in combined data of all 3 breeds. The most significant variant was located at 31,986,084 bp, in an intronic region of the bovine GHR gene (ENSBTAG00000001335). The previously reported F279Y mutation was the second most significant variant (Figure 3).

\section{Haplotype Effects}

Next, we examined haplotype effects at $32,293,167$ bp in HOL, RDC, and JER (Figure $4 \mathrm{~A}-\mathrm{C}$ ). In general, an unfavorable relationship was observed between haplotype effects on milk yield and CM; haplotypes associated with a reduced number of treatments for $\mathrm{CM}$ correlated with decreased milk yield. The most common haplotype in HOL, haplotype HAP47 (frequency $=37.28 \%$ ), had a small favorable effect on milk yield $(5.13 \mathrm{~kg})$ and an unfavorable effect on CM (0.82 clinical cases treated; Figure 4A). Haplotype HAP49 in HOL, which had the highest favorable effect on milk (52.8 $\mathrm{kg})$, had an unfavorable effect on CM (0.96 treatments) 


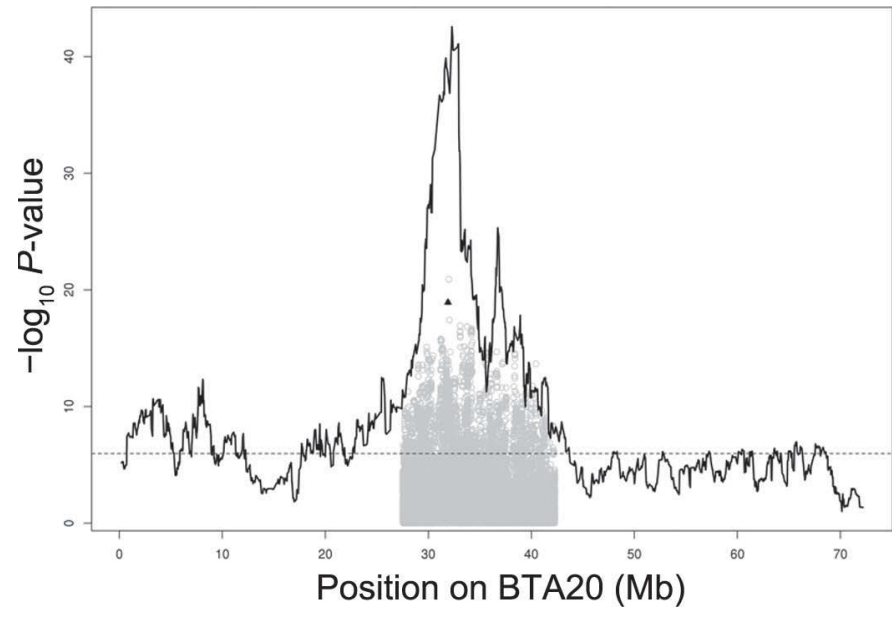

Figure 3. Association mapping of milk QTL on BTA20 using haplotype-based (solid line) and single-marker association analyses (gray circles) in a multibreed combined data set (Holstein, Nordic Red cattle and Jersey). The GHR F279Y mutation is highlighted with a triangle.

and segregated at a moderate frequency (9.78\%). However, some exceptions to this pattern were noted; for example, the second most frequent haplotype, HAP24 in HOL (frequency $=20.3 \%$ ), was the most favorable haplotype for CM ( -2.9 treatments), but had a small favorable effect on milk $(8.18 \mathrm{~kg})$.

We observed a similar pattern in RDC (Figure 4B) and JER (Figure 4C). For example, the most frequent haplotype in RDC, HAP26 (frequency 19.3\%), had a favorable effect on CM (-1.16 treatments) and a small unfavorable effect on milk $(-9.0 \mathrm{~kg})$. Similarly, HAP34 in RDC $(3.83 \%)$ had the highest favorable effect on milk $(83.9 \mathrm{~kg})$, but an unfavorable effect on CM $(1.67$ treatments). Haplotype HAP21 in JER (18.47\%) had a favorable effect on milk $(44.8 \mathrm{~kg})$ and an unfavorable effect on CM (0.76 treatments; Figure 4C).

\section{Inability of the F279Y Mutation in GHR to Explain the Genetic Variance for Milk Yield in HOL}

The F279Y mutation from GHR included as a covariate in the haplotype model only partially explained the QTL effect in the targeted region (Supplementary Figure S4; http://dx.doi.org/10.3168/jds.2015-9599). The most significant SNP associated with milk traits in HOL was located at 30,005,316 bp (rs43140725), close to the mitochondrial ribosomal protein S30 gene (MRPS30, ENSBTAG00000000084, BTA20: 30,072,215-30,081,516 $\mathrm{bp})$. However, on its own, this intergenic variant did not fully explain the full QTL variance (green line in Supplementary Figure S4). Therefore, we tested the effect of the next most significant SNP influencing milk in Holstein, an intronic variant in the protein coding gene BT.89131 (ENSBTAG00000010822) at 33,096,284 bp (rs457676519). This variant failed to explain the QTL variance completely (yellow line in Supplementary Figure S4). Fitting both variants together in the haplotype model also did not explain the QTL variance completely (blue line in Supplementary Figure S4). These results support the hypothesis that not all polymorphisms affecting milk yield in HOL lie within the GHR gene, and that other important genes may be in this area that affect this trait.

\section{Putative Candidate Genes for Clinical Mastitis QTL in HOL}

In our study, CARD6 emerged as a strong candidate gene affecting $\mathrm{CM}$ in HOL. Proteins containing a $C A R D$ region play pivotal roles in signal transduction pathways involved with apoptosis, NF- $\mathrm{B}$ activation, and inflammation (Dufner et al., 2006). The gene CARD6 associates with microtubules and interacts with receptor-interacting protein-like interacting caspase-like apoptosis regulatory protein kinase, a $C A R D$ containing member of the receptor-interacting protein family of protein kinases. These kinases are involved in multiple NF- $\kappa B$ signaling pathways important for innate and adaptive immune responses (Dufner et al., 2006); LIFR also has an anti-inflammatory function (Hunt et al., 2013) and, thus, could play a role in CM susceptibility or resistance.

\section{Putative Candidates Genes for Milk Traits QTL}

The GHR gene emerged as a strong candidate for the regulation of milk yield across all 3 Nordic cattle breeds. This gene encodes a transducer for growth hormone action, which plays a pivotal role in lipid and carbohydrate metabolism (Lucy, 2001). Furthermore, GHR protein has a major role in the growth hormone axis through the initiation and maintenance of lactation (Parmentier et al., 1999), and thus we expected to identify it in our study. Blott et al. (2003) reported a QTL peak that was completely suppressed when the F279Y mutation was included as a fixed effect in an association model. Effects of F279Y on milk, fat, and protein yields and SCS were also reported for German Holstein cattle (Rahmatalla et al., 2011), Jersey (Komisarek et al., 2011), and Fleckvieh (Pausch et al., 2015).

The most significant SNP associated with milk yield in HOL was located at 30,005,316 bp (rs43140725), close to the mitochondrial ribosomal protein MRPS30 gene. However, we found no literature relating this gene to milk production. A previous study (Sadkowski et al., 2009) reported that the expression of MRPS30 in the skeletal muscle was higher $(P<0.001)$ in a beef breed 
(Limousin) than in a dairy breed (HOL). The MRPS30 gene influences cell-mediated immunity and is highly expressed in CD34, B cells, T cells, and natural killer cells (http://biogps.org/\#goto=genereport\&id=10884).

Milk yield traits have an unfavorable genetic correlation with the incidence of CM. Thus, as expected, most haplotypes that we determined to have a favorable effect on milk yield had an unfavorable effect on CM, and vice versa. However, we also identified common haplotypes that affect both milk traits and CM in the same direction (either favorably or unfavorably), as well as haplotypes that exert a large effect on 1 trait but not the other (Figure $4 \mathrm{~A}-\mathrm{C}$ ). Together, these results demonstrate that variations affecting these traits can be separated from each other. Genomic selection, when able to take advantage of this type of information, may
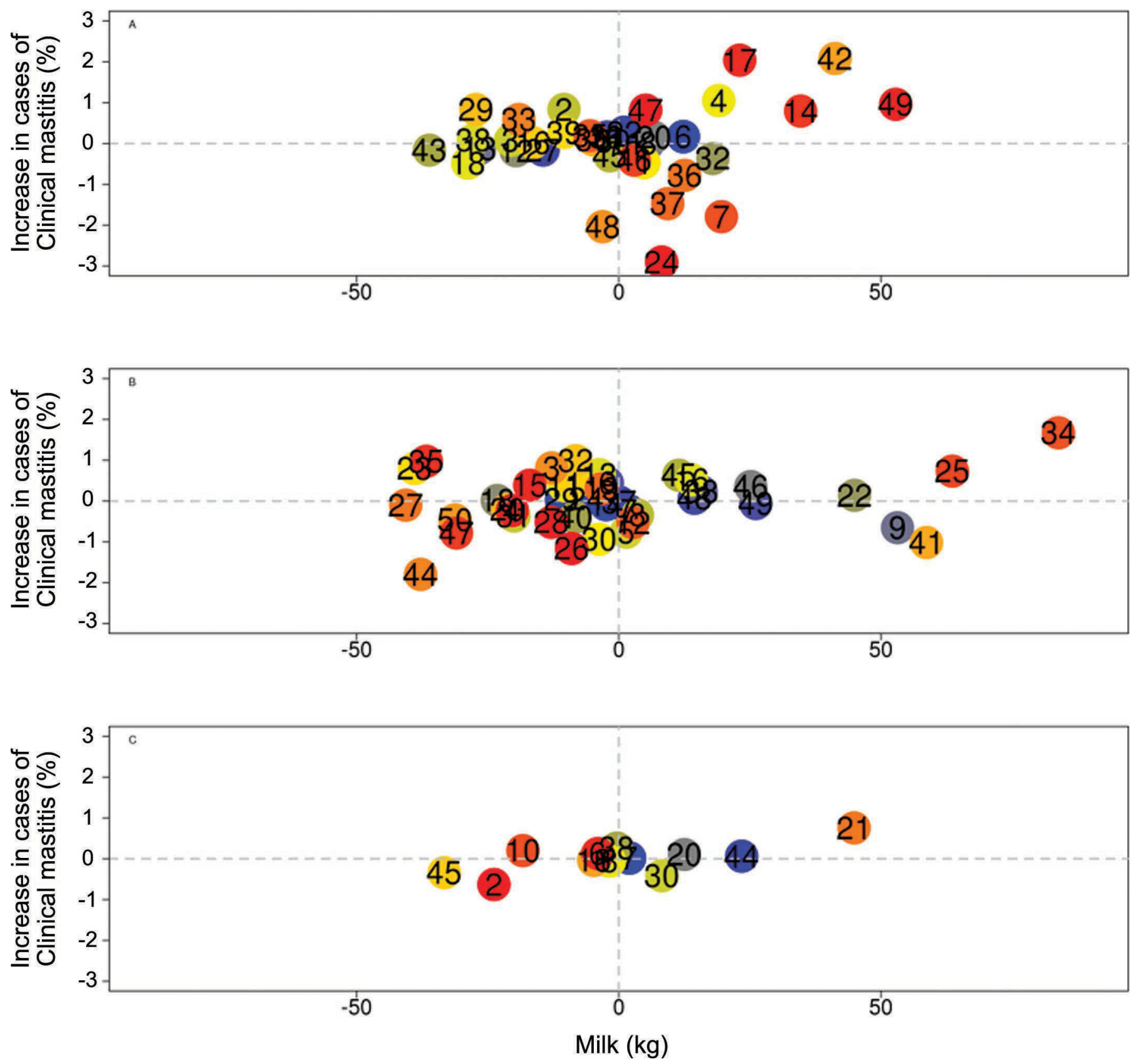

Figure 4. Effect of haplotype on milk (x-axis) and clinical mastitis (y-axis) in Holstein (A), Nordic Red Dairy (B), and Danish Jersey cattle (C). Haplotype states are given for each point. Color indicates the frequency of the haplotypes. Blue, yellow, and red indicate low, medium, and high frequencies, respectively. 
help to reduce the negative effect of the genetic correlation on overall genetic response.

Although we found that the QTL segregate across breeds, the haplotype backgrounds of the QTL alleles were not the same. This fact was evident from the haplotypes showing effects on milk yield traits and $\mathrm{CM}$ that were different in different breeds (Figure 4A-C), even though the haplotype states were inferred jointly for all breeds. Genetic heterogeneity exists across the population where additional causative factor(s) besides the GHR F279Y mutation appear to be segregating. It is possible that these multiple genetic factors have interaction effects (not tested in our study). Such differences in the haplotype background of the QTL and genetic heterogeneity could explain why genomic prediction accuracy is low in across-breed prediction or why very limited improvement in accuracy can be achieved by combing reference populations (Hayes et al., 2013).

We were unable to identify definitively the causal polymorphism(s) affecting $\mathrm{CM}$ and milk traits in the targeted region of BTA20, despite our use of WGS data for multiple breeds of dairy cattle. Several factors may contribute to this difficulty. First, the LD in this region is very high (Supplementary Figure S5; http://dx.doi. org/10.3168/jds.2015-9599); thus, differentiating causal variant(s) from markers in high $\mathrm{LD}$ with the quantitative trait nucleotide(s) was not possible. Second, multiple linked polymorphisms appeared to be affecting 1 or more traits; disentangling their effects was not possible. Third, different causal polymorphisms segregate in different breeds, as evident from the relative haplotype frequencies and their effects. Fourth, it is possible that the causal polymorphisms were not identified in the analyzed panel of SNP because they were removed in the quality control process, segregate at low frequencies (are rare variants), or are structural variants, which are not routinely called by next-generation sequencing.

We observed that additional variants (besides the GHR F279Y mutation) affecting milk yield traits are located on the targeted region on BTA20 in HOL. Milk QTL also segregated in RDC and JER on the BTA20 targeted region. The QTL for CM and milk yield on BTA20 in HOL were due to separate genetic variants. It is possible to select haplotypes having favorable effects on both traits.

\section{ACKNOWLEDGMENTS}

This work was supported by two project grants: (1) Genomic Selection - from function to efficient utilization in cattle breeding (grant no. 3412-08-02253), funded jointly by the Green Development and Dem- onstration Program of the Danish Ministry of Food, Agriculture and Fisheries (Copenhagen, Denmark), the Milk Levy Fund (Aarhus, Denmark), Viking Genetics (Randers, Denmark), and Nordic Cattle Genetic Evaluation (Aarhus, Denmark); and (2) Centre for Genomic Selection in Animals and Plants (GenSAP) funded by The Danish Council for Strategic Research (Copenhagen, Denmark). We are grateful to the Danish Cattle Federation/Nordic Cattle Genetic Evaluation and Viking Genetics for providing the phenotypic data used in this study. The 1,000 Bull Genomes Project (http:// www.1000bullgenomes.com/) is kindly acknowledged for sharing data to impute the genome sequence. Part of the results presented here are part of a patent application number PCT/EP2013/067838 entitled "Genetic markers for mastitis resistance" (AU-2012-634-489). Two anonymous reviewers provided very helpful comments on this manuscript.

\section{REFERENCES}

Bickel, R. D., A. Kopp, and S. V. Nuzhdin. 2011. Composite effects of polymorphisms near multiple regulatory elements create a majoreffect QTL. PLoS Genet. 7:e1001275.

Blott, S., J.-J. Kim, S. Moisio, A. Schmidt-Küntzel, A. Cornet, P Berzi, N. Cambisano, C. Ford, B. Grisart, and D. Johnson. 2003. Molecular dissection of a quantitative trait locus: A phenylalanineto-tyrosine substitution in the transmembrane domain of the bovine growth hormone receptor is associated with a major effect on milk yield and composition. Genetics 163:253-266.

Bohren, B. B., W. G. Hill, and A. Robertso. 1966. Some observations on asymmetrical correlated responses to selection. Genet. Res. 7:44-57.

Brøndum, R. F., B. Guldbrandtsen, G. Sahana, M. S. Lund, and G. Su. 2014. Strategies for imputation to whole genome sequence using a single or multi-breed reference population in cattle. BMC Genomics 15:728.

Brøndum, R. F., G. Su, L. Janss, G. Sahana, B. Guldbrandtsen, D. Boichard, and M. S. Lund. 2015. Quantitative trait loci markers derived from whole genome sequence data increases the reliability of genomic prediction. J. Dairy Sci. 98:4107-4116.

Browning, B. L., and S. R. Browning. 2009. A unified approach to genotype imputation and haplotype-phase inference for large data sets of trios and unrelated individuals. Am. J. Hum. Genet. 84:210-223.

Daetwyler, H. D., A. Capitan, H. Pausch, P. Stothard, R. van Binsbergen, R. F. Brondum, X. Liao, A. Djari, S. C. Rodriguez, C. Grohs, D. Esquerre, O. Bouchez, M. N. Rossignol, C. Klopp, D. Rocha, S. Fritz, A. Eggen, P. J. Bowman, D. Coote, A. J. Chamberlain, C. Anderson, C. P. VanTassell, I. Hulsegge, M. E. Goddard, B. Guldbrandtsen, M. S. Lund, R. F. Veerkamp, D. A. Boichard, R. Fries, and B. J. Hayes. 2014. Whole-genome sequencing of 234 bulls facilitates mapping of monogenic and complex traits in cattle. Nat. Genet. 46:858-865.

de Roos, A., B. J. Hayes, R. Spelman, and M. E. Goddard. 2008. Linkage disequilibrium and persistence of phase in Holstein-Friesian, Jersey and Angus cattle. Genetics 179:1503-1512.

Druet, T., and M. Georges. 2010. A hidden Markov model combining linkage and linkage disequilibrium information for haplotype reconstruction and quantitative trait locus fine mapping. Genetics 184:789-798.

Dufner, A., S. Pownall, and T. W. Mak. 2006. Caspase recruitment domain protein 6 is a microtubule-interacting protein that posi- 
tively modulates NF- $\kappa$ B activation. Proc. Natl. Acad. Sci. USA 103:988-993.

Hayes, B. J., H. A. Lewin, and M. E. Goddard. 2013. The future of livestock breeding: genomic selection for efficiency, reduced emissions intensity, and adaptation. Trends Genet. 29:206-214.

Heringstad, B., Y. M. Chang, D. Gianola, and G. Klemetsdal. 2005. Genetic association between susceptibility to clinical mastitis and protein yield in Norwegian dairy cattle. J. Dairy Sci. 88:1509-1514.

Heringstad, B., G. Klemetsdal, and J. Ruane. 2000. Selection for mastitis resistance in dairy cattle: A review with focus on the situation in the Nordic countries. Livest. Prod. Sci. 64:95-106.

Howie, B. N., P. Donnelly, and J. Marchini. 2009. A flexible and accurate genotype imputation method for the next generation of genome-wide association studies. PLoS Genet. 5:e1000529.

Hunt, L. C., A. Upadhyay, J. A. Jazayeri, E. M. Tudor, and J. D. White. 2013. An anti-inflammatory role for leukemia inhibitory factor receptor signaling in regenerating skeletal muscle. Histochem. Cell Biol. 139:13-34.

Komisarek, J., A. Michalak, and A. Walendowska. 2011. The effects of polymorphisms in DGAT1, GH and GHR genes on reproduction and production traits in Jersey cows. Anim. Sci. Pap. Rep. 29:29-36.

Li, H., and R. Durbin. 2009. Fast and accurate short read alignment with Burrows-Wheeler transform. Bioinformatics 25:1754-1760.

Li, H., B. Handsaker, A. Wysoker, T. Fennell, J. Ruan, N. Homer, G. Marth, G. Abecasis, and R. Durbin. 2009. The sequence alignment/map format and SAMtools. Bioinformatics 25:2078-2079.

Lucy, M. C. 2001. Reproductive loss in high-producing dairy cattle: Where will it end? J. Dairy Sci. 84:1277-1293.

Lund, M. S., J. Jensen, and P. Petersen. 1999. Estimation of genetic and phenotypic parameters for clinical mastitis, somatic cell production deviance, and protein yield in dairy cattle using Gibbs sampling. J. Dairy Sci. 82:1045-1051.

Lund, M. S., A. P. Roos, A. G. Vries, T. Druet, V. Ducrocq, S. Fritz, F. Guillaume, B. Guldbrandtsen, Z. Liu, R. Reents, C. Schrooten, F. Seefried, and G. Su. 2011. A common reference population from four European Holstein populations increases reliability of genomic predictions. Genet. Sel. Evol. 43:43.

Lund, M. S., G. Sahana, L. Andersson-Eklund, N. Hastings, A. Fernandez, N. Schulman, B. Thomsen, S. Viitala, J. L. Williams, A. Sabry, H. Viinalass, and J. Vilkki. 2007. Joint analysis of quantitative trait loci for clinical mastitis and somatic cell score on five chromosomes in three Nordic dairy cattle breeds. J. Dairy Sci. 90:5282-5290.

Madsen, P., and J. Jensen. 2010. DMU, A package for analysing Multivariate Mixed Models. Version 6, release 5.0. Accessed July 2013. http://dmu.agrsci.dk/DMU/Doc/Current/dmuv6_guide.5.2.pdf.

McKenna, A., M. Hanna, E. Banks, A. Sivachenko, K. Cibulskis, A. Kernytsky, K. Garimella, D. Altshuler, S. Gabriel, and M. Daly. 2010. The Genome Analysis Toolkit: A MapReduce framework for analyzing next-generation DNA sequencing data. Genome Res. 20:1297-1303.

Oltenacu, P. A., and D. M. Broom. 2010. The impact of genetic selection for increased milk yield on the welfare of dairy cows. Animal Welfare 19:39-49.

Parmentier, I., D. Portetelle, N. Gengler, A. Prandi, C. Bertozzi, L. Vleurick, R. Gilson, and R. Renaville. 1999. Candidate gene markers associated with somatotropic axis and milk selection. Domest. Anim. Endocrinol. 17:139-148.

Pausch, H., C. Wurmser, F. Reinhardt, R. Emmerling, and R. Fries. 2015. Short communication: Validation of 4 candidate causative trait variants in 2 cattle breeds using targeted sequence imputation. J. Dairy Sci. 98:4162-4167.

Price, A. L., N. J. Patterson, R. M. Plenge, M. E. Weinblatt, N. A. Shadick, and D. Reich. 2006. Principal components analysis corrects for stratification in genome-wide association studies. Nat. Genet. 38:904-909.

Rahmatalla, S. A., U. Müller, E. M. Strucken, M. Reissmann, and G. A. Brockmann. 2011. The F279Y polymorphism of the GHR gene and its relation to milk production and somatic cell score in German Holstein dairy cattle. J. Appl. Genet. 52:459-465.

Sadkowski, T., M. Jank, L. Zwierzchowski, J. Oprzadek, and T. Motyl. 2009. Comparison of skeletal muscle transcriptional profiles in dairy and beef breeds bulls. J. Appl. Genet. 50:109-123.

Sahana, G., B. Guldbrandtsen, B. Thomsen, L. E. Holm, F. Panitz, R. F. Brøndum, C. Bendixen, and M. S. Lund. 2014. Genomewide association study using high-density single nucleotide polymorphism arrays and whole-genome sequences for clinical mastitis traits in dairy cattle. J. Dairy Sci. 97:7258-7275.

Sahana, G., B. Guldbrandtsen, B. Thomsen, and M. S. Lund. 2013. Confirmation and fine-mapping of clinical mastitis and somatic cell score QTL in Nordic Holstein cattle. Anim. Genet. 44:620-626.

Sahana, G., M. S. Lund, L. Andersson-Eklund, N. Hastings, A. Fernandez, T. Iso-Touru, B. Thomsen, S. Viitala, P. Sørensen, and J. Williams. 2008. Fine-mapping QTL for mastitis resistance on BTA9 in three Nordic red cattle breeds. Anim. Genet. 39:354-362.

Schulman, N. F., G. Sahana, T. Iso-Touru, M. S. Lund, L. AnderssonEklund, S. M. Viitala, S. Varv, H. Viinalass, and J. H. Vilkki. 2009. Fine mapping of quantitative trait loci for mastitis resistance on bovine chromosome 11. Anim. Genet. 40:509-515.

Schulman, N. F., G. Sahana, T. Iso-Touru, S. D. McKay, R. D. Schnabel, M. S. Lund, J. F. Taylor, J. Virta, and J. H. Vilkki. 2011. Mapping of fertility traits in Finnish Ayrshire by genome-wide association analysis. Anim. Genet. 42:263-269.

Sherry, S. T., M.-H. Ward, M. Kholodov, J. Baker, L. Phan, E. M. Smigielski, and K. Sirotkin. 2001. dbSNP: The NCBI database of genetic variation. Nucleic Acids Res. 29:308-311.

Sodeland, M., H. Grove, M. Kent, S. Taylor, M. Svendsen, B. J. Hayes, and S. Lien. 2011. Molecular characterization of a long range haplotype affecting protein yield and mastitis susceptibility in Norwegian Red cattle. BMC Genet. 12:70.

USDA. 2008. Dairy 2007 Part III. Reference of Dairy Cattle Health and Management Practices in the United States 2007:154. USDAAnimal and Plant Health Inspection Service-Veterinary Service, Center for Epidemiology and Animal Health, Fort Collins, CO.

van Binsbergen, R., R. F. Veerkamp, and M. P. L. Calus. 2012. Makeup of the genetic correlation between milk production traits using genome-wide single nucleotide polymorphism information. J. Dairy Sci. 95:2132-2143.

Yu, J., G. Pressoir, W. H. Briggs, I. Vroh Bi, M. Yamasaki, J. F. Doebley, M. D. McMullen, B. S. Gaut, D. M. Nielsen, J. B. Holland, S. Kresovich, and E. S. Buckler. 2006. A unified mixed-model method for association mapping that accounts for multiple levels of relatedness. Nat. Genet. 38:203-208.

Zhang, Z., M. Erbe, J. L. He, U. Ober, N. Gao, H. Zhang, H. Simianer, and J. Q. Li. 2015. Accuracy of whole-genome prediction using a genetic architecture-enhanced variance-covariance matrix. G3 (Besthesda) 5:615-627. 10.1534/g3.114.016261.

Zimin, A. V., A. L. Delcher, L. Florea, D. R. Kelley, M. C. Schatz, D. Puiu, F. Hanrahan, G. Pertea, C. P. Van Tassell, and T. S. Sonstegard. 2009. A whole-genome assembly of the domestic cow, Bos taurus. Genome Biol. 10:R42. 